\title{
Challenges of Numerical Simulation of Dynamic Wetting Phenomena: A Review
}

\author{
Shahriar Afkhami \\ Department of Mathematical Sciences, New Jersey Institute of Technology, Newark, NJ, \\ USA 07102
}

\begin{abstract}
Wetting is fundamental to many technological applications that involve the motion of the fluid-fluid interface on a solid. While static wetting is well understood in the context of thermodynamic equilibrium, dynamic wetting is more complicated in that liquid interactions with a solid phase, possibly on molecular scales, can strongly influence the macroscopic scale dynamics. The problem with continuum models of wetting phenomena is then that they ought to be augmented with microscopic models to describe the molecular neighborhood of the moving contact line. In this review, widely used models for the computation of wetting flows are summarized first, followed by an overview of direct numerical simulations based on the Volume-of-Fluid approach. Recent developments in the Volume-of-Fluid simulations of the wetting are then reviewed, with a particular attention paid on combining macro-scale simulations with the hydrodynamic theory near the moving contact line, as well as including a microscopic description by coupling with the van der Waals interface model. Finally, the extension to modeling the contact line motion on non-flat surfaces is surveyed, followed by hot topics in nucleate boiling.
\end{abstract}

Keywords:

\section{Introduction}

Wetting phenomena involves the motion of the interface between two or more fluids in contact with a solid [1. Many natural and industrial processes are intimately related to the details of wetting phenomena. As technological capability evolves, it becomes increasingly important to understand the fundamentals of the fluid dynamical interactions involved in such applications, in order to optimize system performance. Numerical modeling offers great potential to predict outcomes under given conditions, and hence, ultimately, to optimize the process. Moreover, computational tools can play a very important role in terms of

\footnotetext{
* Corresponding author

Email address: shahriar.afkhami@njit.edu (Shahriar Afkhami)
} 
enhancing our understanding of the physics involved, and allowing predictions to be made cheaply.

Simulating wetting phenomena is complicated due to the presence of moving 'contact lines' (the lines at which a fluid-fluid interface meets a solid surface) [2 6]. Considerable research effort has been directed to study such problem; see e.g. [7, 8] for a comprehensive (and relatively recent) reviews. Simulating such phenomena is further complicated, in part, by the fact that the description of moving contact lines involves widely varying characteristic lengths 9 - 12, from macroscopic to near molecular length scales. The motion of the fluid near the contact line and the dynamic behavior of the interface itself present further complications, and analytical solutions exist only for very simple problems. Numerical simulations can yield accurate predictions but have been far less successful when studying wetting phenomena. For such problems, the efficacy of numerical simulation is highly dependent on the ability of accurately and robustly computing the moving contact line.

Another potential issue is that at the contact line, as the characteristic length of the problem becomes smaller and the surface tension starts to dominate over other forces, the surface tension discretization becomes a key ingredient in the feasible computation of such flows. For these reasons, though reliable codes exist for the simpler two dimensional problem, there are relatively few examples of three dimensional codes that incorporate algorithms for treating dynamic contact lines; 13 19] are representative of the variety of approaches that are used. Moreover, robust and accurate numerical models are needed for simulating fluid-structure interactions that involve moving solid objects in an interfacial flow that also includes contact lines (see e.g. [18, 20]).

While there exist different numerical approaches which can deal with dynamic contact lines, here we concentrate on the 'Volume-of-Fluid' (VOF) method, with our main goal to be to represent recent developments for the implementation of the contact angle boundary condition at a moving contact line, selfconsistent numerical validations, a dynamic contact angle model based on a hydrodynamic description of the contact line, as well as an approach for the direct inclusion of liquid-solid interaction. We specifically pursue Gerris [21] numerical framework to describe the implementations of the methods. The interested reader is referred to the works in $22+24$ for further details and discussions. However, we also note possibilities of using other tools than Gerris, such as the use of its successor code Basilisk [25]; see e.g. 19, 26, 27] for very recent studies that utilize Basilisk for the numerical simulation of dynamic contact line. While the focus of this review is on a VOF method, we note that a major challenge for the simulation of moving contact lines in all continuum based approaches is to obtain mesh-independent results with no adjustable parameters. The development of such models will require resolving all the length scales, typically from nanoscale at the solid boundary to bulk sizes, which can be of a millimeter size or more. For feasible computations therefore sub-grid models ought to be introduced, and as a result, the key ingredient in all current and previous models is to bridge the macroscale dynamics to the characteristics of the contact line at the nanoscale. 
The nature of flows in a neighborhood of a moving contact line and the matching of local solutions to the global flow have been the topic of a number of investigations; see e.g. [28, 29]. Despite the huge research effort, however, a full understanding of the mechanism by which a contact line moves along a solid surface is still incomplete. While numerical investigations can enhance our understanding of the physics involved, simulating dynamic contact line flows is complicated by the mathematical paradox of a contact line moving along a no-slip solid surface, first discussed by Huh and Scriven in [30. Here we will review major difficulties involved in continuum-level numerical simulations in the VOF numerical framework, mainly: (i) how to specify the dynamic contact angle; and (ii) how to modify the no-slip boundary condition to remove the stress singularity [6, 31].

We first give a concise account of a variety of other computational methods that have been considered in the context of wetting phenomena. Here we mention level set methods that use a continuous function to describe the interface. A reconstruction of the continuous function in the ghost domain is used to impose the contact angle within the level set approach [3, 32, 33]. (The grid points across the boundary are treated as ghost points). We also mention phase-field methods that treat two fluids with a diffuse interface by means of a smooth concentration function, which typically satisfies the Cahn-Hilliard or Allen-Cahn equations 34, and is coupled to the Navier-Stokes equations. Jacqmin [35] describes a phase-field contact angle model that uses a wall energy to determine the value of the normal derivative of the concentration on a solid substrate. This model has been used to study contact line dynamics 2, 36, and similar models have been considered in the investigation of the sharp interface limit of the diffuse interface model [37, 38. Lattice-Boltzmann methods have also treated the contact angle with a wall energy contribution [39, 40]. Molecular dynamics (MD) simulations [41,44] have also been considered, where the interaction between fluid and solid particles is described by the Lennard--Jones potential, albeit mainly for simulating nanoscale systems.

In [45], the authors carry out continuum based computations of dewetting of molten nanoparticles on a solid and show quantitative agreement with MD simulations, when either using a free-slip boundary condition, or a partial slip with a slip length of nanometer sizes. In [46, the authors carry out simulations of steady water drop sheared between two moving plates using MD, phasefield, and VOF methods. They show discrepancy, when comparing the results from phase-field to MD simulations. Moreover, they show that with a suitable choice of parameters and boundary conditions in the phase-field simulations, a reasonable agreement with MD can be obtained. They also reproduce the MD results reasonably well with the VOF simulations using a localized slip boundary condition. Interestingly, they point out the role of diffusion in their phase-field model and the physical interpretation of it, showing different effect to what happens in MD simulations, suggesting a perfect agreement cannot be expected. In summary, the authors in [46] point out the necessity for hybrid methods for matching MD with continuum solvers, similar in spirit to that in [47. 
Finally, in the context of front-tracking methods, in [48, the authors implement a front-tracking method and use a partial slip boundary condition and an ad hoc model for the determination of the dynamic contact angle based on the contact line speed. Recently, in [16, the authors developed a front-tracking approach for simulating dynamic contact angles, where the contact line motion is described by a slip model.

In what follows, we first present in Sec. 2, a multiscale wetting/dewetting model based on an adaptive VOF method coupled with van der Waals interaction, which can be considered as a mesoscopic approach to model liquid-solid interaction. We then review a work on wetting transitions in Sec. 3 . In Sec. 4. we discuss an approach to model the contact line motion on non-flat surfaces and its application to simulating a two-phase flow in porous media. Lastly, in Sec. 5. as an example of a future direction, we present nucleate boiling, where resolving the details of the flow around a moving contact line is of a significant importance to building a generally applicable numerical model.

\section{A mesoscopic approach to model liquid-solid interaction}

It is impossible for a contact line to move on a solid if a molecularly sharp fluid-fluid interface is considered along with a strictly no-slip solid. The consequence of such is the divergence of shear stress upon approaching the contact line. As elegantly stated by Pomeau in [10, "One major difficulty met when trying to 'solve' the moving contact line problem is to find at which scale the usual continuum mechanics breaks down and should be amended to get rid of the divergence.". Here, we review a recently developed computational scheme for wetting/dewetting flows, that combines the van der Waals model with the Navier-Stokes equations to devise a divergence free numerical framework for the simulation of contact line motion 49 . We show how this approach can lead to accurate description of the contact line, while recovering the usual macroscopic scale flow far away from the contact line.

Let us begin by considering the incompressible Navier-Stokes equations for a two-phase flow. We refer to two phases as the liquid phase (subscript $l$ ), and the vapor phase (subscript $v$ ), i.e. $i=l$, $v$, with no loss of generality. We introduce a characteristic function $\chi(x, y, z, t)$, which takes the value of 1 inside of the liquid phase, and 0 inside the vapor phase. The interface between these two phases is assumed to be sharp, so that $\chi$ changes discontinuously at the interface. The governing equations then are

$$
\begin{gathered}
\rho(\chi) \frac{D \mathbf{u}}{D t}=-\nabla p+\nabla \cdot\left[\mu(\chi)\left(\nabla \mathbf{u}+\nabla \mathbf{u}^{\top}\right)\right]+\gamma \kappa \mathbf{n} \delta_{s}+\mathbf{F}_{v d W}, \\
\nabla \cdot \mathbf{u}=0 .
\end{gathered}
$$

Here, $\rho(\chi)=\rho_{l} \chi+\rho_{v}(1-\chi)$ is the density, $\mu(\chi)=\mu_{l} \chi+\mu_{v}(1-\chi)$ the viscosity, $p$ the pressure, and $\mathbf{u}$ the velocity vector. Also

$$
D \chi / D t=\partial_{t} \chi+\mathbf{u} \cdot \nabla \chi=0 .
$$




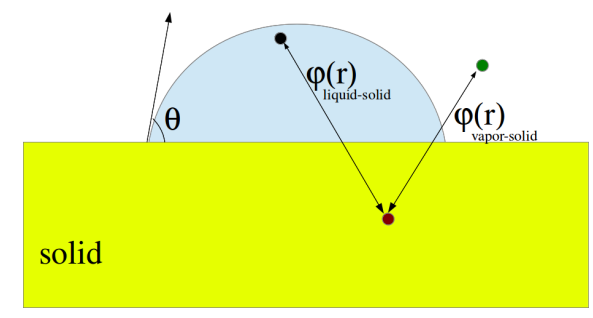

Figure 1: A schematic of the fluid-fluid-solid interactions.

The surface tension is included as a singular body force (per unit volume) 24, 50, where $\gamma$ is the surface tension coefficient, $\kappa$ the interfacial curvature, $\delta_{s}$ a delta function centered on the interface, and $\mathbf{n}$ a normal vector for the interface pointing out of the liquid. The total body force due to the van der Waals interaction is represented by $\mathbf{F}_{v d W}$, which we describe next.

We consider a solid phase occupying a half-infinite region $y<0$, above which there is a region occupied by two fluids, see Fig. 11. Each particle of fluid phase $i$ interacts with the solid substrate by means of a Lennard-Jones type potential [11, 12]

$$
\phi_{i s}=K_{i s}\left(\left(\frac{\sigma}{r}\right)^{a}-\left(\frac{\sigma}{r}\right)^{b}\right),
$$

where $r$ is the distance between the two particles, and $K_{i s}$ is the scale of the potential. The total potential energy in phase $i$ due to this interaction is

$$
\Phi_{i s}(y)=\mathcal{K}_{i s}\left[\left(\frac{h}{y}\right)^{m}-\left(\frac{h}{y}\right)^{n}\right]=\mathcal{K}_{i s} \psi(y),
$$

where

$$
\begin{gathered}
\mathcal{K}_{i s}=2 \pi n_{i} n_{s} K_{i s} \sigma^{3}\left(\frac{[(a-2)(a-3)]^{b-3}}{[(b-2)(b-3)]^{a-3}}\right)^{\frac{1}{a-b}}, \\
h=\left[\frac{(a-2)(a-3)}{(b-2)(b-3)}\right]^{\frac{1}{a-b}} \sigma ; \quad m=a-3, \quad n=b-3,
\end{gathered}
$$

where $n_{i}$ and $n_{s}$ are the densities of particles in fluid phase $i$ and the solid substrate, respectively. Equation 5 gives the total potential per unit volume in fluid phase $i$ due to the interaction with the solid substrate. The quantity $h$ is conventionally referred to as the equilibrium film thickness in the literature. The parameters $m$ and $n$ are taken based on the choices of $a$ and $b$ in Eq. 4. see [49, 51, for example.

The force per unit volume on phase $i$ as a results of the potential, Eq. 5 , is $\mathbf{F}_{i s}(y)=-\nabla \Phi_{i s}(y)$, where

$$
\mathbf{F}_{v d W}=\chi \hat{\mathbf{F}}_{l s}+(1-\chi) \hat{\mathbf{F}}_{v s}
$$




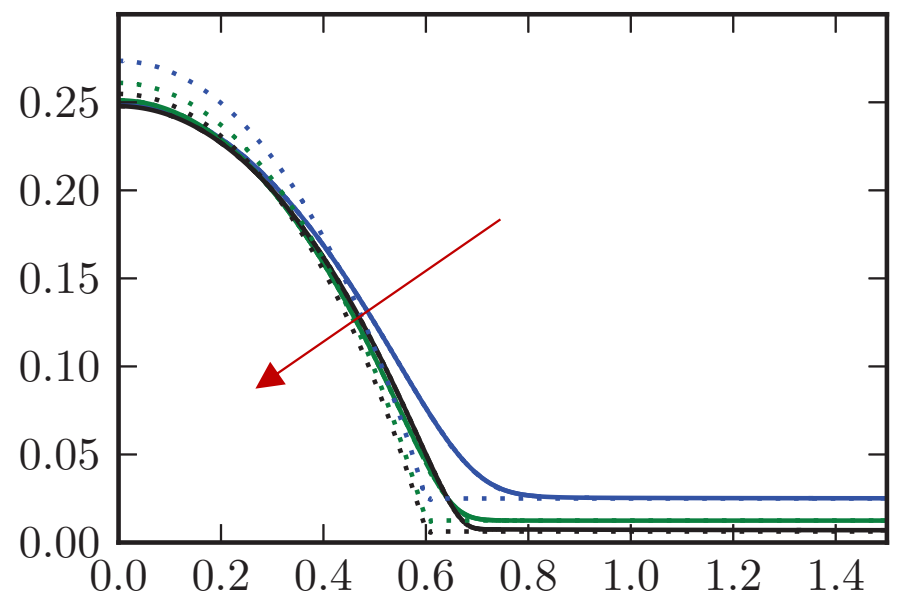

Figure 2: Equilibrium profiles (solid lines) for $\theta_{i}=\theta_{\mathrm{eq}}=\pi / 4$; the arrow shows the direction of decreasing $h$. The dotted lines show the initial profile. $h=0.025$ (blue), 0.0125(green), 0.00625 (black);

It can then be shown [49] that

$$
\mathbf{F}_{v d W}=\nabla p_{v d W}+\frac{\left(1-\cos \theta_{\mathrm{eq}}\right)}{h} \frac{(m-1)(n-1)}{m-n} \psi(y) \mathbf{n} \delta_{s} .
$$

The first term on the right hand side of Eq. 9 is absorbed into the pressure gradient in Eq. 1 for the entire domain, while the second term is centered only on the interface. We refer the interested readers to 24] for the details of the discretization of $\kappa$ in Eq. 1 , and $\mathbf{n}$ and $\delta_{s}$, in Eqs. 1 and 9 .

The coefficient in the second term on the right hand side of Eq. 9 involving the equilibrium contact angle $\theta_{\text {eq }}$ can be derived based on simple energetic arguments (see [49]). In this context, there is an equilibrium film of thickness $h$ that wets the entire substrate, with a smooth transition from the interface away from the contact to the film. The contact angle can then be defined by finding the slope of the interface at the transition region. For example, for a drop at equilibrium with a vanishingly small $h$, we measure contact angles by fitting a circular profile to the droplet profile 'away' from the transition region; the angle at which this circular profile intersects with the equilibrium film is taken to be the contact angle, $\theta_{\text {eq }}$. For non-vanishing but small $h$, a drop at equilibrium will have a (slightly) different contact angle, which we refer to as $\theta_{\text {num }}$. Figure 2 shows how the simulated equilibrium contact angle, $\theta_{\text {num }}$, is generally smaller than the imposed angle $\theta_{\text {eq }}$. This is due to the fact that Eq. 9 is derived under the assumption of small $h$. Figure 2 also shows the results when $h$ is varied from 0.025 to 0.00625 . The initial condition is imposed with $\theta_{i}=\pi / 4$. As shown, the initial drops, depicted by the black (dotted) profiles, relax to the equilibrium profiles, and as $h$ is decreased, the equilibrium profiles are characterized by contact angles closer to prescribed by $\theta_{\text {eq }}$. 


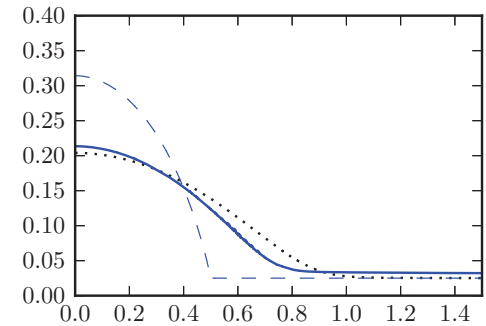

(a)



(c)

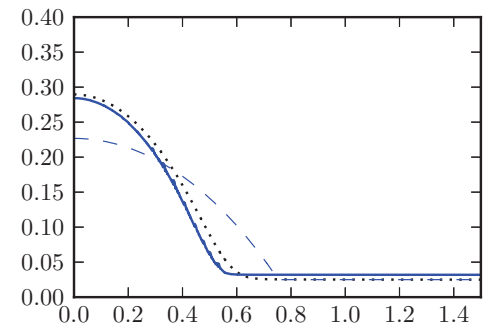

(b)

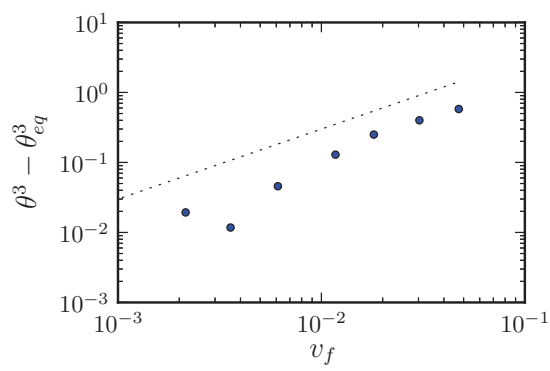

(d)

Figure 3: (a) Spreading of drop from initial contact angle $\theta_{i}=\pi / 3$ to equilibrium defined by $\theta_{\mathrm{eq}}=\pi / 6$ and (b) retraction from $\theta_{i}=\pi / 6$ to $\theta_{\mathrm{eq}}=\pi / 3$. Comparison with the Cox-Voinov law for (c) spreading and (d) retracting drop. The blue (symbols) shows simulation results and the black (dashed) line is proportional to $v_{f}$, showing the expected slope if the Cox-Voinov [52, 53] law is obeyed.

\subsection{Results}

We present the results of droplets with the contact angles imposed by means of the discretization of Eq. 9 as described in [49, 51. The initial simulation setup is a drop on an equilibrium film, initially at rest, which then relaxes to equilibrium under the influence of the van der Waals force. For all the results shown next, $\rho_{l, v}=\mu_{l, v}=\gamma=1, h=0.025$, and $(m, n)=(3,2)$. Figures 3(a-b) show the results of droplet spreading and retracting. As shown, the droplet spreads/retracts to its equilibrium configuration, defined by the $\theta_{\text {eq }}$. We also compare the qualitative behavior of the spreading/retracting drop to the well known Cox-Voinov law [53. For a drop displacing another immiscible fluid on a solid surface, the speed of the contact point, $v_{f}$, is related to instantaneous angle $\theta$ and the equilibrium contact angle $\theta_{\text {eq }}$ to the leading order by $\theta^{3}-\theta_{\text {eq }}^{3} \propto$ $v_{f}$ [52. As shown, the drop spreading/retracting follows the Cox-Voinov law for $v_{f} \in\left(10^{-2}, 10^{-1}\right)$.

Finally, we mention the dewetting phenomena of liquid films: for a sufficiently thin liquid layer (or multi-layers) on a solid substrate, destabilizing effects, such as long range fluid-solid interactions, can lead to the film breakup 

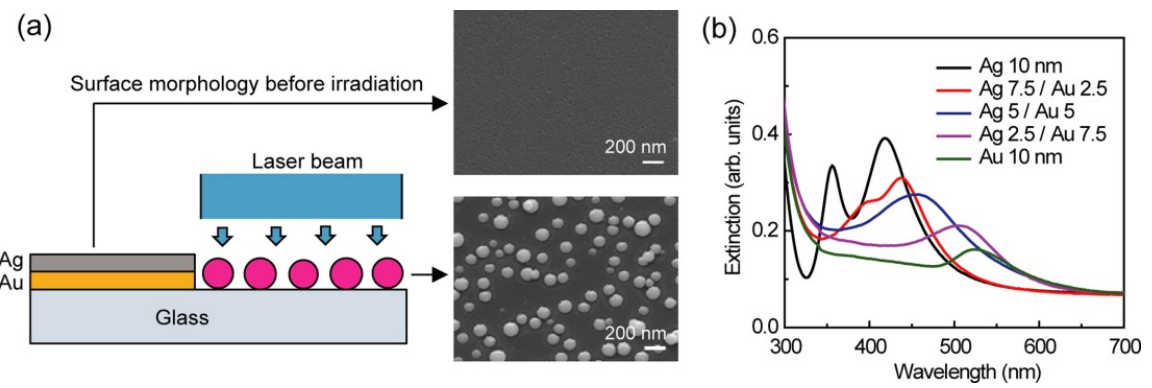

Figure 4: From 58. (a) Morphologies of a bilayer thin film before and after laser irradiation. (b) Extinction spectra measured after thins films of different layer combination are dewetted.

and the consequent dewetting phenomena (exposure of prewetted/nominally dry patches), known as 'spinodal dewetting' [54]. Therefore, it is necessary to include a destabilizing mechanism, such as the van der Waals interactions as described above, or otherwise a continuous film does not breakup. While studying the dewetting of a thin film is commonplace, see e.g. [55] 57], there is less effort in direct numerical simulations of multi-layer dewetting, and the understanding of competing forces for such systems is currently poor. Below, we describe our recent results of the dewetting of miscible liquid two-layers on substrates. In particular, we mention the work in [58] on the fabrication of $\mathrm{Ag}-\mathrm{Au}$ bimetallic nanoparticles by laser-induced dewetting of bilayer films; see for example, Fig. 4. Next we show examples of direct numerical simulations to study the structural evolution and morphology of bilayer thin films consisted of miscible liquids. We will concentrate in particular on the role of the contact angle and the viscosity ratio of the two layers to study how changing these properties may affect the profile of the fluid as it dewets. We keep the geometrical features fixed, and set the density ratio and surface tension to 1 , with a negligibly small surrounding viscosity. Figure 5 depicts the schematic of the computational setup. We then vary the contact angle and the liquid two-layer viscosity ratio and consider two main scenarios where the breakup of the film results in either an unchanged length scale, namely leading to the formation of one drop, or 'dry spots', leading subsequently to the formation of one main drop and a secondary droplet. The findings here will help to understand how to control the synthesis of alloy metallic nanoparticles, see e.g. [59]. Basilisk [25], which is the Gerris successor numerical framework, is used here. We have specifically found: 1) In the absence of a second layer, the instability of the thin film follows closely the predictions of the linear stability analysis [51; see Fig. 6(a). 2) By making the bottom layer slightly more viscous than the top layer, while the bilayer still remains unstable, the nonlinear instability leads to the formation of a secondary droplet, suggesting that even a small spatial variation of the viscosity can significantly alter the instability characteristic length scales; see Fig. 6(b). 3) There appears to be a correlation between the viscosity ratio and contact angle defining the threshold for the formation of a secondary droplet; see Fig. 7 . 


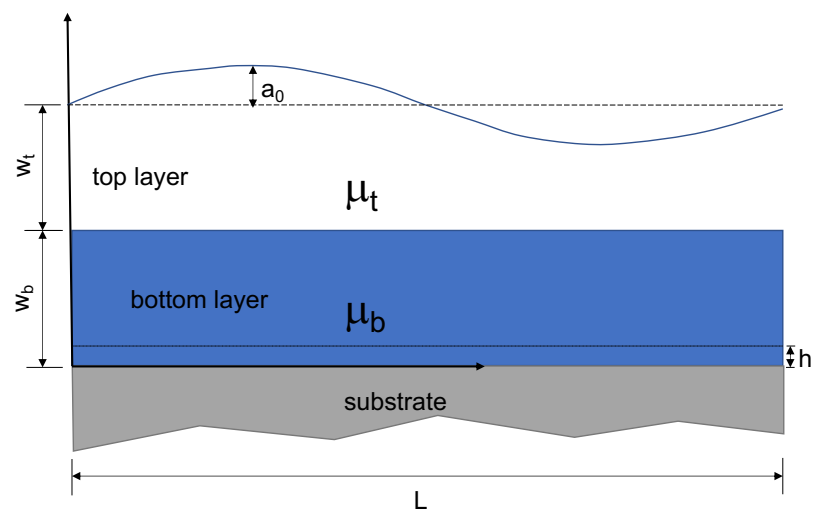

Figure 5: Schematic of the computational setup. Viscosity ratio is $\mu_{b} / \mu_{t}, L$ is the wavelength of a linearly unstable sinusoidal wave with an amplitude $a_{0}$, and $h$ is the prewetted or precursor film thickness.



(a)

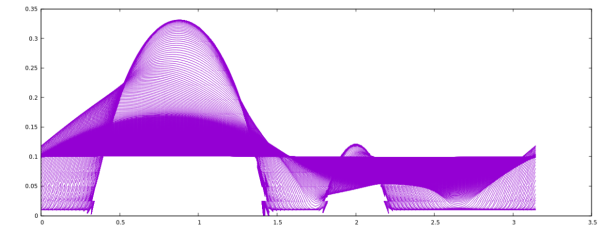

(b)

Figure 6: Formation of a single drop (a) and two drops (b). $L=\pi, w_{b}=w_{t}=0.05, h=0.01$, $a_{0}=1 \mathrm{e}-3, \theta=90^{\circ}$, and (a) $\mu_{b} / \mu_{t}=1$ (b) $\mu_{b} / \mu_{t}=1.1$.

Our future work will involve investigating film lengths, layer sequences, and the influence of the surrounding viscosity. We will also further study the compositional structure of the bilayer systems. Our preliminary numerical results reveal that the obtained final droplets have a composition of the two layers over the whole volume of the droplets; while our results show qualitatively similar distribution as illustrated in the experiments in [58, further analysis of the differences in the composition as a function of the physical parameter set will help tuning the morphology of such nanostructures for applications.

\section{A multiscale model for the simulation of dynamic contact lines: the dewetting transition}

Here we review a recent work in [6] on the description of a method for dynamic contact lines. The work focuses on in-depth analysis on the physical 


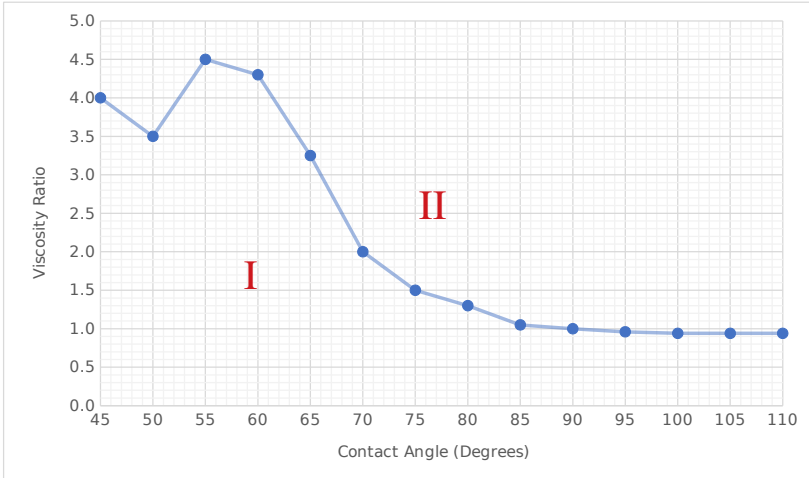

Figure 7: The threshold for the formation of the secondary droplet (symbols), showing the viscosity ratio $\mu_{b} / \mu_{t}$ as a function of the contact angle $\theta$. The geometrical parameters are the same as in Fig. 6. Formation of a single drop (I) and two drops (II).

phenomenon of the dewetting transition. When modeling dynamic contact line, here in the context of the VOF method, there are two major challenges: 1) the numerical implementation of the contact angle, and 2) relieving the incompatibility between the moving contact line and the no-slip boundary condition (see e.g. 60, for a recent mathematical view of the boundary conditions for dynamic wetting). In [6], the authors address both difficulties by first describing a method to impose the contact angle as a boundary condition for when discretizing the surface tension in computational cells adjacent to the solid boundary, and secondly, by devising an elaborate methodology to account for microscopic features near the contact line, using an asymptotic theory for the solution of the local contact line problem and to remove the singularity in the shear stress, leading to numerical convergence. We note however that a fundamental question still remains regarding the measurement of the microscopic contact angle and its definition at a dynamic contact line, see e.g. 61 for a recent and renewed discussion on the topic, and review papers [8, 54,62 .

The discretization of Eq. 3 consists of two steps; first the reconstruction of the interface followed by its advection. In the first part of the reconstruction step, the interface normal $\mathbf{n}=\left(n_{x}, n_{y}\right)$ in cel is determined from the values $\chi$ in neighboring cells, using method described in 4, 23. In the reconstruction step, the position of a linear segment representing the interface in the cell is determined from the knowledge of $\mathbf{n}$ and $\chi[63$. The reconstructed linear segments are updated in the advection step, where the interface is evolved by the fluid velocity field, using Eq. 3 .

In order to compute capillary forces, we use the Height-Function method, in which the local height of the interface is computed from summing over a column of cells [4. Using second-order finite differences of the local Height- 




Figure 8: Schematic illustrating the withdrawing plate setup.

Function then provides the curvature, as well as the interface normals, used to compute the surface tension force (see [23, 24]). Near the contact line, the interface is then linearly extended into the solid cell to prescribe the value of $\chi$ function at ghost cells. Having the interface Heights (and Widths) now defined at ghost cells, interface normal vector and curvature adjacent to the solid boundary are computed as usual. Once the interface positions and the curvature are computed, there is no special difficulty in computing the velocity field using the standard methods. No special provision is made for the discontinuity of velocities or the divergence of viscous stresses and pressures, which are computed as elsewhere in the domain using finite volumes and finite differences. Intuitively, this allows a kind of numerical slip. This numerical slip is studied in details in [5].

\subsection{Results}

The problem setup is as follows: consider a solid plate being withdrawn from a liquid reservoir with a constant velocity $V_{s}>0$. The computational domain is $0 \leq x, y \leq L$, with fluid 1 occupying $y<h_{0}$ and fluid 2 occupying $y>h_{0}$ at $t=0$ (see Fig. 8). The viscosity and density of fluid $i=1,2$ are $\mu_{i}$ and $\rho_{i}$, respectively. The capillary number is defined as $\mathrm{Ca}=\mu_{1} V_{s} / \sigma$, where $\mu_{i}$ is the viscosity of fluid $i$ and $\sigma$ the surface tension. We set $L \approx 9 l_{c}$, where $l_{c}$ is the capillary length $l_{c}=\sqrt{\sigma /\left[\left(\rho_{1}-\rho_{2}\right) g\right]}$ with $g$ the gravitational acceleration. The Reynolds number is then defined based on the capillary length as $\operatorname{Re}=\rho_{1} V_{s} l_{c} / \mu_{1}$.

In [6], we focus on a specific, complex physical problem: the dewetting transition. One example of such a flow is the withdrawing plate, see Fig. 8 The interface may either sustain a stationary state meniscus, if below a critical capillary number, $\mathrm{Ca}_{c r}$, or continue to move up the substrate until depositing a thin film to arbitrary heights. The latter is called a Landau-Levich-Derjaguin (LLD) film 64, 65. Figure 9 from [6] shows an example of the case where $\mathrm{Ca}>\mathrm{Ca}_{c r}$. In 66 68, a hydrodynamic theory is developed for the prediction of $\mathrm{Ca}_{c r}$, while Cox [52] and Voinov [53] describe, on the other hand, how the 


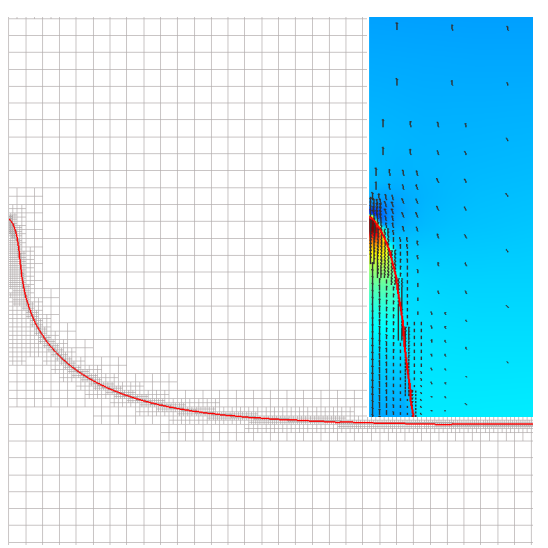

(a)

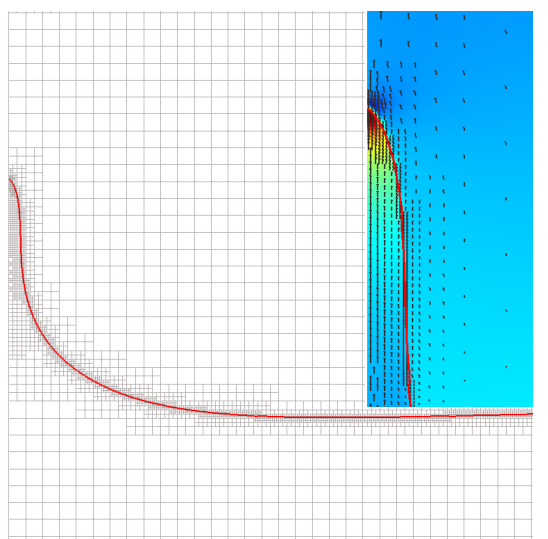

(b)

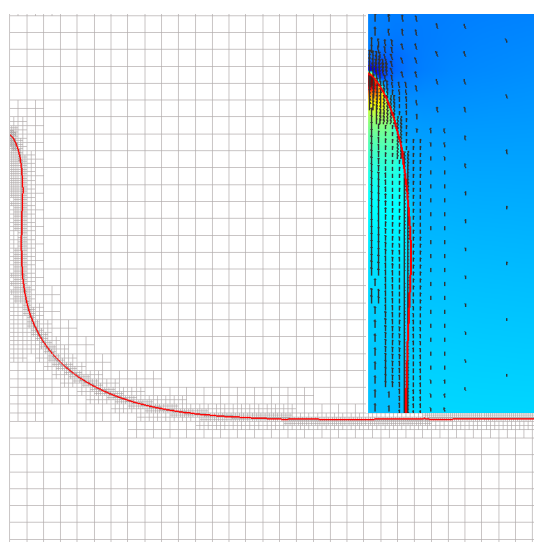

(c)
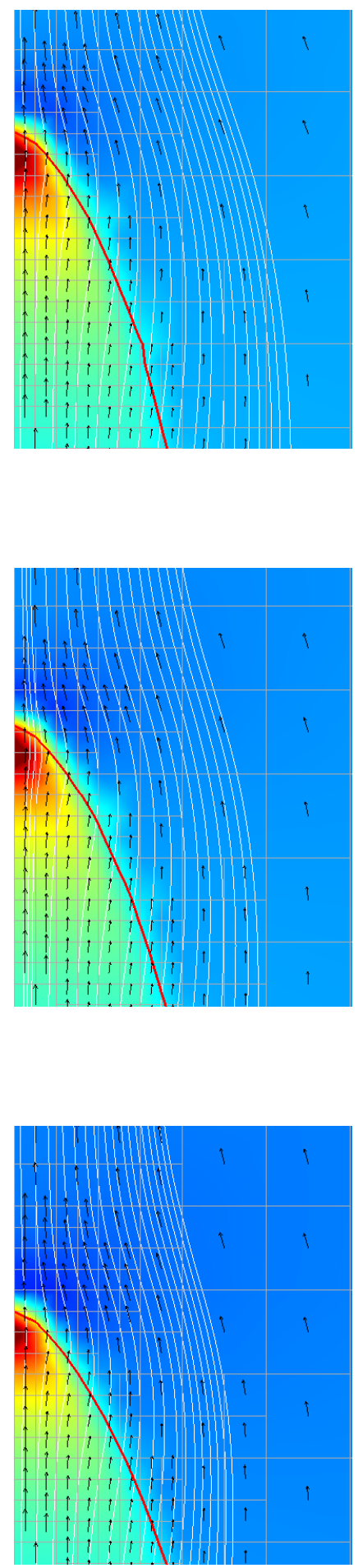

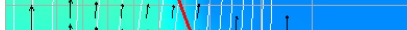




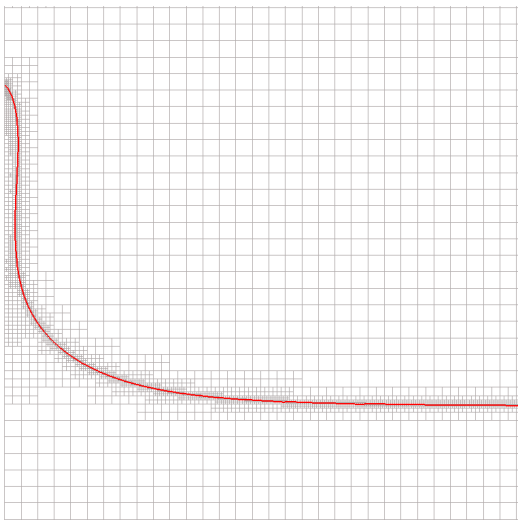

(d)

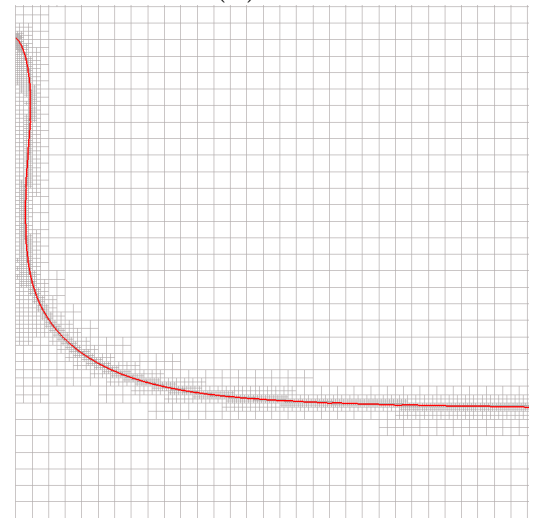

(e)

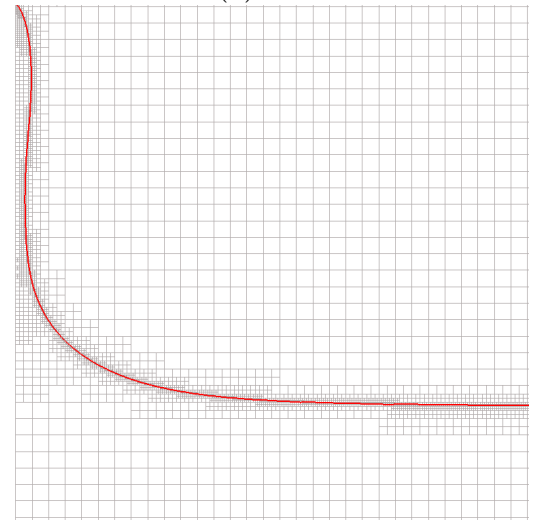

(f)

Figure 9: From 6. Time evolution of the interface for $\mathrm{Ca}>\mathrm{Ca}_{c r}$ at $\tau=V_{s} t / l_{c}=4$ (a), 4.8 (b), 5.9 (c), 6.8 (d), 7.9 (e), 8.7 (f). The insets show the magnified flow field and the pressure distribution. (a)-(c) Right panels show a magnified view of the contact line region and the computational mesh; the fine structure of the flow field and the pressure distribution in the contact line region are illustrated. $\mathrm{Ca}=0.048$, and $\theta=60^{\circ}$. The pressure colors show the maximum (dark red) and minimum (dark blue) of the pressure distribution. 
singularity drives a peculiar curved form of the fluid wedge at small Ca. In [6], we use these theories to predict the numerically observed transition and to devise a subgrid scale model to reproduce the underlying microscopic physics. The latter reflects on the notion of grid-independent simulations in [5, 69. In the simulations, we measure $\mathrm{Ca}_{c r}$ and specify $\Delta$, the typical grid size, and $\theta_{\Delta}$, the specified contact angle at a particular grid size $\Delta$. We compare the value of $\mathrm{Ca}_{\mathrm{cr}}$ from full simulations to the solutions of the central relation obtained in [6. 70

$$
\frac{C(q) \phi\left(\theta_{\Delta}, q\right) \mathrm{Ca}_{c r}^{1 / 3} l_{c}}{\Delta} \exp \left[-\frac{G\left(\theta_{\Delta}\right)}{\mathrm{Ca}_{c r}}\right]=1,
$$

where the constant $C(q)=\mathcal{O}(1), q=\mu_{2} / \mu_{1}, G$ function is given in [52, and $\phi=\Delta / r_{m}$ is the scaling factor or gauge function determined from the numerical simulations by comparing to the analytical solutions, where we consider $r_{m} \propto \Delta$, namely the numerical slip. In [6, 70, the computed $C a_{c r}$ are in agreement with the above solutions. Finally, it can be shown that

$$
\phi\left(\theta_{\Delta}, q\right)=\frac{\pi \mathrm{eAi}^{2}\left(s_{\max }\right)}{3^{1 / 3} 2^{-5 / 6}} \frac{\Delta}{\mathrm{Ca}_{c r}^{1 / 3} l_{c}} \exp \left[\frac{G\left(\theta_{\Delta}, q\right)}{\mathrm{Ca}_{c r}}\right],
$$

where $\operatorname{Ai}(\mathrm{x})$ is the Airy function of the first kind and $\mathrm{Ai}^{\prime}\left(\mathrm{s}_{\max }\right)=0$; see also an earlier work in [71, where an expression similar to Eq. 11 is derive.

In Fig. 10, we plot the values of the right hand side of Eq. 11 along with the computed values of $\phi$. In the near-free-surface Setup C $(q=1 / 50)$, a very clean estimate of the gauge function $\phi$ is obtained at small angles and we find $\phi\left(\theta_{\Delta}\right) \simeq \theta_{\Delta}$. Thus our numerical model may be viewed as having an effective slip of the order of a grid cell. However, we find that there is a mismatch between the theory and the data in Eq. 11 in Fig. 10. If one leaves the connection between the slip length $\lambda$ and the grid size $\Delta$ free, using $\lambda=c \Delta$ with $c$ an arbitrary constant, then the adjustable constant $c$ allows a better fit of to the data; see, for example, the dashed line (--) in Fig. 10 for when choosing $c=3$. This in turn provides an estimate of the effective slip in our VOF method.

\subsubsection{Recommendations for the numerical simulations of dynamic contact lines}

As we discussed above, numerical slip plays a crucial role in numerical simulations of the moving contact line problem. As shown, this numerical slip, which is introduced at the discrete level, is effectively equivalent to a slip length on the order of the grid size. Several authors have reported how this numerical slip can strongly distort the results [5, 72, 73, unless the grid size in the vicinity of the contact line is decreased to a microscopic scale, making the computations prohibitively costly.

To develop a strategy for realistic simulations where experiments would be used, the first step would be to perform a series of simulations at varying $\Delta$ and for the conditions of the experiments. Obviously, simulations and experiments are not performed near the critical $\mathrm{Ca}$ but below it. Comparing the angles observed in the region where the theory is still valid, would cross-validate the 


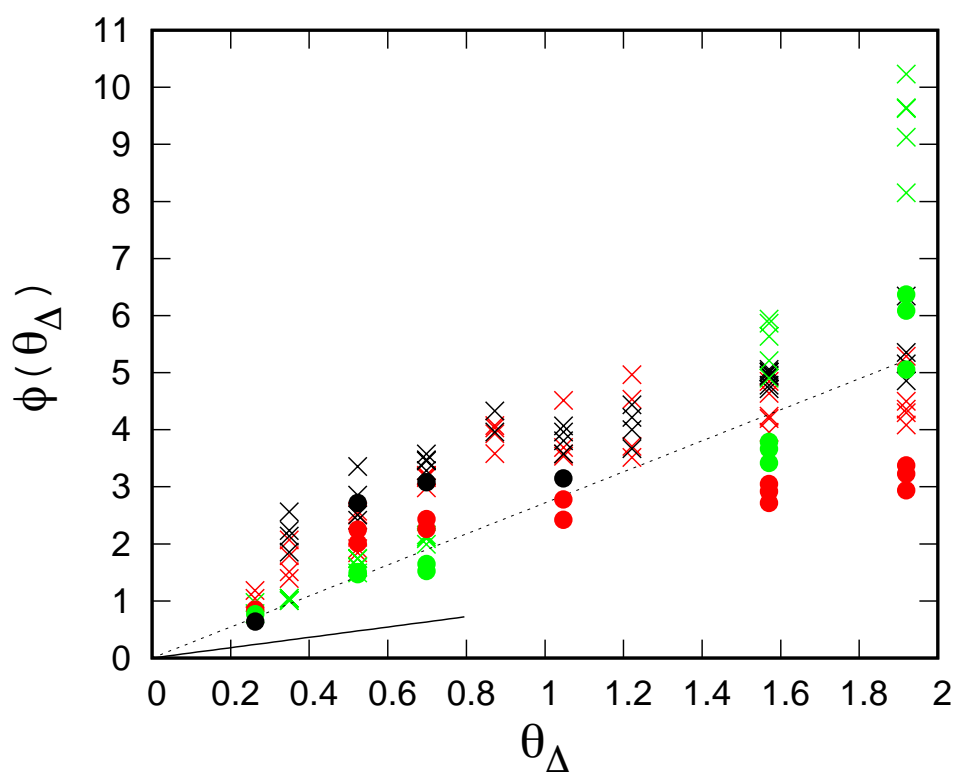

Figure 10: Using the data from 6, 70, $\phi$ is plotted using expression Eq. 11 for Setups A: $q=1$ $(\times)$, B: $\operatorname{Re}=q=1(\times)$, and $\mathrm{C}: q=1 / 50(\times)$, compared to the computed values from the best fit of $\phi$ for Setups A $(\bullet), \mathrm{B}(\bullet)$, and C $(\bullet)$, with $\theta_{\Delta}$ measured in radian. For a given angle $\theta_{\Delta}$, the various values of $\phi$ correspond to the various values of the grid size $\Delta$ for each data set. For all the simulations, the density ratio $\rho_{1} / \rho_{2}=5$. The solid line $(-)$ is the prediction from lubrication theory for $\phi=\mathrm{e} \theta_{\Delta} / 3$, when assuming $\lambda \approx \Delta$ and dashed line (- -), is for when adjusting $\lambda \approx 3 \Delta$.

computations. This would in turn fix the parameters $\theta_{\Delta}$ and $r_{m}$ that could be used in simulations. This approach is of course made difficult by the fact that there is no evidence so far that a single pair $\theta_{\Delta}, r_{m}$ could predict experiments over a range of different flow configurations. Actually the authors of [74 state that "In the literature we have not found a single pair of experiments in different geometries using precisely the same materials.". This highlights the difficulty of a predictive simulation approach based on experiments. Moreover, it should be noted that the authors of references [75] and [76] claim precisely the opposite, that no microscopic angle, even a dynamical one depending on the capillary number, can predict the whole range of experiments they have performed or simulated. We therefore believe that the relationship between numerical slip length and the grid size is strongly problem dependent. We provide such a relation in Eq. 11 for the withdrawing plate problem in the hope that it motivates further investigations for other scenarios, such as droplet impact on a solid surface, where such relationships can also involve other relevant dimensionless parameters such as Reynolds and Weber numbers. A suitable numerical slip can then be identified by comparing the results with appropriate experimen- 
tal measurements. We note however again that such a relation might only be applicable to that particular problem.

Finally, the slip boundary condition combined with a constant contact angle might not sufficiently describe the dynamics of the moving contact line. In [26, the authors propose to replace the slip boundary condition with a generalized Navier boundary condition (GNBC) [77, coupled with a dynamic contact angle to more favorably match the experimental observations in the context of the curtain coating configuration [75] (see also [60, 78, for a recent discussion on the boundary conditions for dynamic wetting).

\section{Moving contact lines on arbitrary geometries}

Numerically modeling non-ideal solid substrate is enormously complicated. Wetting and spreading on rough surfaces, two-phase flow over topographically patterned surfaces and through porous media are examples of such systems. While there exist numerical studies on chemically disordered surfaces, see e.g. 79, 80, and with roughness effects in a two-phase flow model, see e.g. 81 83, porescale numerical investigations are still scarce, see e.g. [84 86, and therefor in what follows, we only focus on the direct numerical simulation of fluid-fluid flows in a porous media model.

Pore-scale models have increasingly become a reliable tool for making predictions of two-phase flows through porous media; see e.g. 87, 88. Direct numerical simulation techniques based on solving the full Navier-Stokes equations provide an accurate solution for pore-scale analysis of multiphase flows in porous media. However, a great challenge in pore-scale direct numerical simulations is the inclusion of wetting effects into the numerical model. There exist direct numerical studies on pore-scale modeling of wettability effects, such as the phase-field simulation in [89] and the lattice Boltzmann simulation in 90. While the majority of previous (Eulerian) interface capturing methods include wetting on body-fitted meshes, less progress has been made to develop numerical methods that handle arbitrary geometries (see e.g. [32]). To that end, one attractive approach is the Immersed Boundary Method (IBM) 91, 92, because of its ability to model complex geometries, and its potential for modeling systems of solid bodies with arbitrary relative motion [20]. Here we show an accurate and robust numerical model developed in 93 and later extended in 20] for combining wetting dynamics, in a VOF framework, and the IBM. Here we briefly review the methodology developed in 93 as the basis of an IBM for implementing wetting effects into the numerical model for arbitrary geometries.

The method developed in 93 is based on a ghost-cell Immersed Boundary (IB) method for curved surfaces. In order to compute the interface curvature in cells adjacent to an IB computational cell, the authors employ a method where the IB stencil is modified to take into account the orientation of the interface normal at the contact line to reflect the prescribed contact angle. The modified stencil is illustrated in Fig. 11. At each point where the stencil intersects the IB, the prescribed contact angle defines the value of the interface normal at the contact line, $\overrightarrow{\mathbf{n}}_{c l}$. Therefore, adjacent cells to the IB enter into the computation 


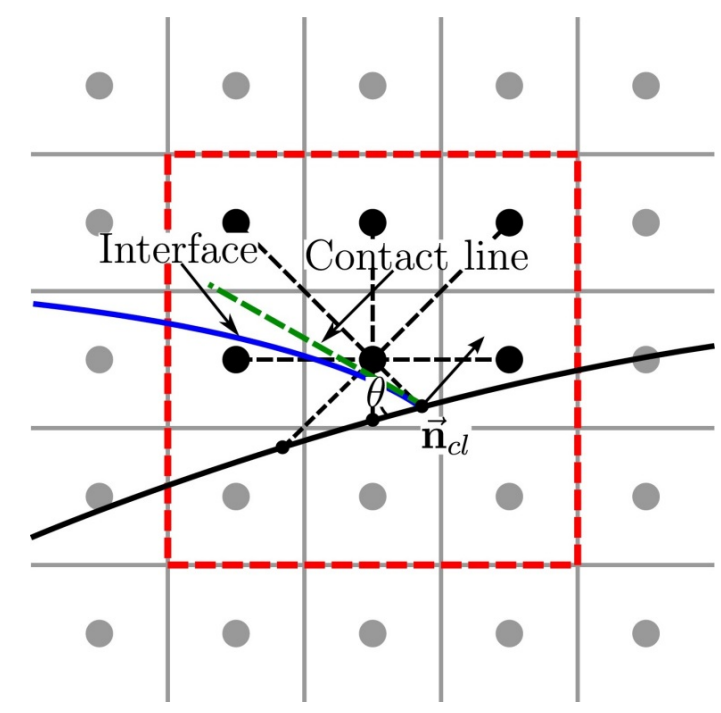

Figure 11: From 93. The stencil used for the evaluation of curvature, $\kappa$, near the IB surface, the normal to the interface at the contact line is determined from the prescribed contact angle.

of the curvature, $\kappa$, resulting in a wetting force that consequently enters the surface tension discretization.

In 94, we present the results of an improved implementation of the above method 20] to simulate two-phase flows in a porous media model. The results are first of their kind in that they are obtained using a combined VOF/IBM pore-scale direct numerical simulations to quantify the effects of the capillarity, characterized by the capillary number, $\mathrm{Ca}$, and wetting, characterized by the contact angle, $\theta$, on the displacement phenomena in a porous media model. In Fig. 12, we show how wetting controls displacement patterns, and observe the crossover from a stable propagating front to a fractal pattern, namely a fingering instability. The results show that decreasing the contact angle leads to a change of the flow behavior from strong fingering to stabilized front propagation, and that the contact angle effect diminishes as Ca increases. As can be seen in Fig. 12 displacement patterns, for wetting invading fluids, the front propagates rather smoothly, draining all of the displaced phase, while a non-wetting invading fluid can lead to residual fluid.

\section{Future direction: microlayer formation in nucleate boiling as an example}

Pool boiling is one of the most efficient mode of heat transfer, allowing a wide range of systems to improve their thermal performance, from nuclear power 


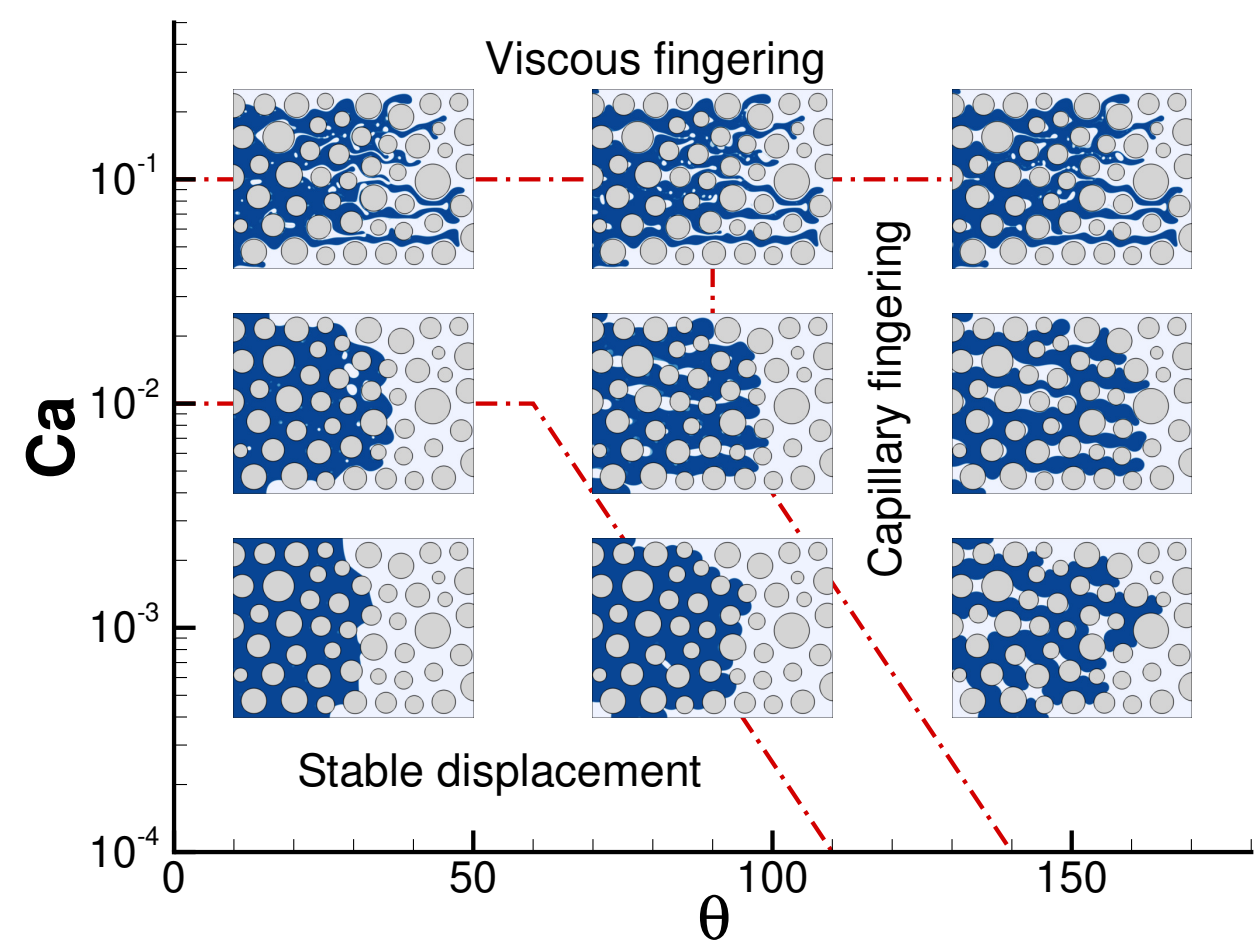

Figure 12: From 94. Displacement patterns at varying $\mathrm{Ca}$ and $\theta$. Above a certain Ca threshold only viscous fingering is visible for the parameters studied in this work.

plants to microelectronic devices [95. Predicting boiling heat transfer however is complicated by the need to resolve phenomena occurring over multiple scales (see Fig 13), from the adsorbed liquid layer at the wall at the nanometer scale up to the bubble size at the millimeter scale. Furthermore, a significant amount of the total heat can be transferred within the micro-region (known as microlayer) near the contact line. Existing models of microlayer formation are often physically incomplete, e.g. do not include the effect of surface tension or contact angle. Moreover, the dynamics of the moving contact line has a profound influence on the microlayer formation. Therefore, a general model on microlayer formation must also resolve the motion of the contact line.

In [96, the authors present experimental and numerical observations of contact line heat transfer in the framework of pool boiling and meniscus evaporation. They focus on the influence of contact line dynamics on the local heat transfer near the contact line suggesting that the heat transfer next to the contact line is governed mainly by two mechanisms in superposition: microlayer evaporation and transient conduction. In [97, the authors present a numerical 


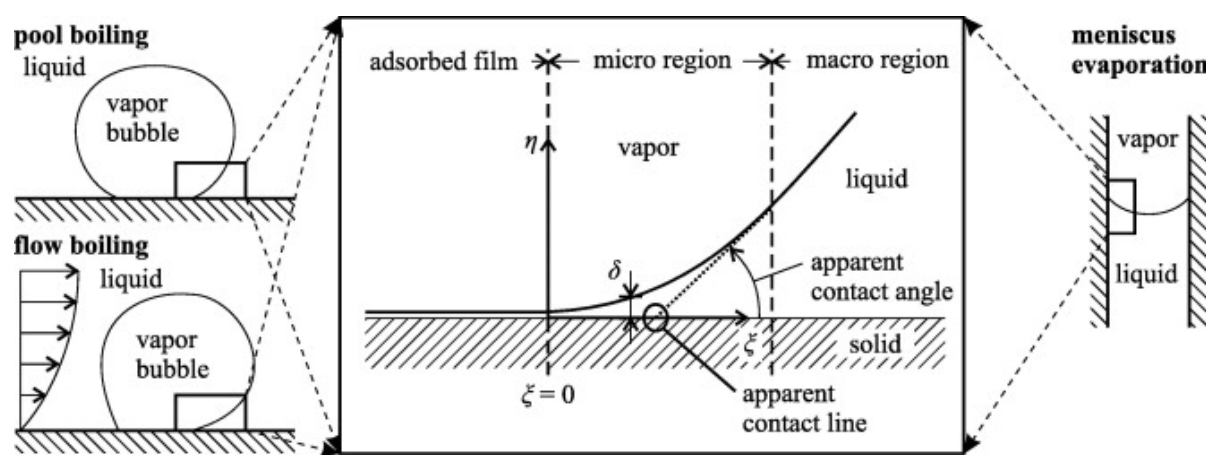

Figure 13: From 96]. Multiple scales involved in nucleate boiling: from the millimeter scale at the bubble cap to the nanometer scale at the adsorbed liquid layer.

framework in which a subgrid source of vapor is considered. In [98, the authors present experimental results on the transition between contact line evaporation and microlayer evaporation during the dewetting of a superheated wall. They discuss the time resolved formation of the microlayer in detail and describe the influence of the dewetting velocity, wall superheat, and heating power on the microlayer formation. In [99, the authors describe the microlayer formation as a dewetting transition in the presence of phase change and use the existing theoretical, experimental and numerical data to a derive a specific criterion for modeling the transition.

Here, we provide a review of the work in 97 of the procedure for direct computations of nucleate boiling and the conditions under which the microlayer is expected to form. In [97, we focus on numerical simulation of the hydrodynamics of bubble growth at a wall, and high resolution simulations of the microlayer formation. We identify the minimum set of dimensionless parameters that controls microlayer formation, namely $\delta^{*}=f\left(r^{*}, t^{*}, \mathrm{Ca}, \theta\right)$ with $\delta^{*}$ the shape of the extended liquid microlayer, $r_{c}=\mu_{l} /\left(\rho_{l} U_{b}\right)$ and $t_{c}=r_{c} / U_{b}$ the characteristic length and time scales used, respectively, where $U_{b}$ is the bubble growth rate. We found that all three regions need to be modeled in order to accurately represent the thickness of the microlayer. Central to our theory is the results that the interface profile in the microlayer away from the contact line can be accurately described (in the case of hemispherical bubble growth) as $\delta^{*} \approx 0.5 \sqrt{r^{*}-R_{b, 0}^{*}}$ as long as the motion of the contact line is negligible compared to the bubble growth rate; $R_{b, 0}^{*}$ is the dimensionless initial bubble radius and $r^{*}$ is the dimensionless radial distance from the bubble root.

Experimental measurements of the earliest bubble growth rate (Fig. 14 (a)) and microlayer thickness (Fig. 14 (b)) are then used in [97 to validate the numerical results, for the case of water at saturation at $0.101 \mathrm{MPa}$. The numerical results are compared with experimental results in Fig. 14 (b), showing both qualitative and quantitative agreements. However, a departure from the 


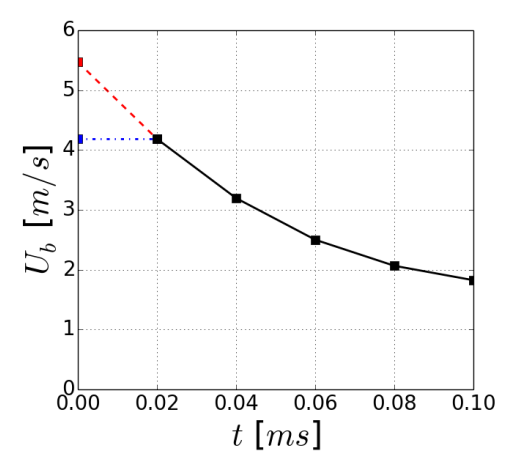

(a)

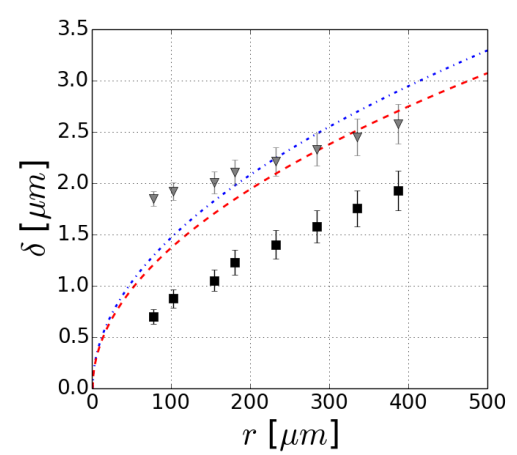

(b)

Figure 14: From: 97. Measured bubble growth rates (black squares) over time (a); the earliest bubble growth rate is measured at $t=20 \mu \mathrm{s}$, showing that the initial bubble growth rate during the first tens of $\mu s$ may have been much higher than its first available measured value of $4.2 \mathrm{~m} / \mathrm{s}$. Two scenarios assumed for initial regimes: constant growth rate (blue dashdotted line), or constant acceleration (red dashed line). Thickness $\delta$ (in $\mu m$ ) measured in lab experiment (black squares) of boiling water at saturation at $0.101 M P a, 0.41 \mathrm{~ms}$ after boiling inception. The deduced microlayer thickness at $t=20 \mu \mathrm{s}$ (grey triangles) is obtained assuming that the heat transfer within the microlayer is purely by conduction. The wall temperature at boiling inception is $111.7^{\circ} \mathrm{C}$, and the wall heat flux is $209 \mathrm{~kW} / \mathrm{m}^{2}$. Predictions from the square root model are plotted for the two initial profiles of $U_{b}$ showed in (a). The first measured value of $U_{b}$ is $U_{b}(t \sim 20 \mu \mathrm{s}) \sim 4.2 \mathrm{~m} / \mathrm{s}$. For simulations: $\mu_{l}=3.5 \times 10^{-4} \mathrm{~Pa} . \mathrm{s}, \rho_{l}=950 \mathrm{~kg} / \mathrm{m}^{3}$.

model in the low thickness region is also observed. This discrepancy is expected to be reduced if we no longer assume constant wall superheat, but include the thermal coupling between the microlayer and the heated wall. Such coupling would require to solve for the heat equation in the substrate. The initial evaporation of the microlayer would locally reduce the wall superheat and therefore slow down the rate of evaporation of the microlayer. This slow-down effect of the microlayer evaporation due to conjugate heat transfer with the solid surface is currently not included in the results in Fig. 14, and must be a subject of further investigation. In general, systematic experiments seem to be necessary to validate the numerical model and to develop more applicable dynamic contact line models.

\subsection{Hot topics and future issues}

Numerical challenges for dynamic contact lines amplify when including heat and mass transfer mechanisms (i.e. evaporation, boiling, and condensation). The numerical framework described in this review will face new challenges to accurately and robustly implement the velocity discontinuity across the interface due to the mass exchange. Including the conjugate heat transfer with the solid substrate, which requires the coupling of the flow solver and the energy 
equations, as well as a domain decomposition strategy, introduces additional challenges; see e.g.[100. Here, we list some future issues:

- The continuum formulations for moving contact line models lay out a strong foundation for studying such systems. We believe that more elaborate models that include microscopic phenomena are needed to calibrate and verify numerical results towards building a more robust moving contact line model.

- A static contact angle is not a compatible model to impose on a moving contact line. We believe a dynamic contact angle model should address a more physically correct model of the contact line, which is essential for simulating real applications.

- Hybrid numerical models that include hydrodynamic theories of the dynamic contact line show promise. A more general theories to large contact angles are needed to extend the applicability of such hybrid models. Moreover, a special attention should be given on the development of numerical methods that include more level of details, still maintaining computational efficiency.

- Extension of all the above items to textured surfaces poses a formidable numerical challenge.

- This review provides an example of a powerful numerical tool, Gerris, for studying relevant problems. While laying out a strong foundation, more improved numerical methods should continue to be developed for increasing the predictive power of direct simulations for real life engineering applications, especially for three-dimensional domains.

\section{Acknowledgment}

We acknowledge the support by the Petroleum Research Fund PRF-59641ND. We thank P. Lehrer for help with Basilisk computations, K. Mahady, for fluid-fluid-solid interaction simulations, and S. Zaleski for fruitful discussions and help with improving this review.

[1] P. de Gennes, Wetting: Statics and Dynamics, Rev. Mod. Phys. 57 (1985) 827.

[2] J. Jacqmin, Contact-line dynamics of a diffuse fluid interface, J. Fluid Mech. 402 (2000) 57.

[3] P. D. Spelt, A level-set approach for simulations of flows with multiple moving contact lines with hysteresis, J. Comput. Phys. 207 (2005) 389.

[4] S. Afkhami, M. Bussmann, Height functions for applying contact angles to 2D VOF simulations, Int. J. Numer. Meth. Fluids 57 (2008) 453. 
[5] S. Afkhami, S. Zaleski, M. Bussmann, A mesh-dependent model for applying dynamic contact angles to VOF simulations, J. Comput. Phys. 228 (2009) 5370.

[6] S. Afkhami, J. Buongiorno, A. Guion, S. Popinet, Y. Saade, R. Scardovelli, S. Zaleski, Transition in a numerical model of contact line dynamics and forced dewetting, J. Comput. Phys. 374 (2018) 1061.

[7] Wörner, M., Numerical modeling of multiphase flows in microfluidics and micro process engineering: a review of methods and applications, Microfluidics Nanofluidics 12 (2012) 841.

[8] Y. Sui, H. Ding, P. D. M. Spelt, Numerical simulations of flows with moving contact lines, Annu. Rev. Fluid Mech. 46 (2014) 97.

[9] L. M. Pismen, Y. Pomeau, Disjoining potential and spreading of thin liquid layers in the diffuse-interface model coupled to hydrodynamics, Phys. Rev. E 62 (2000) 2480.

[10] Y. Pomeau, Recent progress in the moving contact line problem: a review, Comptes Rendus Mécanique 330 (2002) 207.

[11] L. M. Pismen, B. Y. Rubinstein, Spreading of a wetting film under the action of van der Waals forces, Phys. Fluids 12 (2000) 480.

[12] V. Starov, V. Kalinin, J.-D. Chen, Spreading of liquid drops over dry surfaces, Adv. Colloid Interface Sci. 50 (1994) 187.

[13] M. Bussmann, J. Mostaghimi, S. Chandra, On a three-dimensional volume tracking model of droplet impact, Phys. Fluids 11 (1999) 1406.

[14] H. Ding, P. D. M. Spelt, Wetting condition in diffuse interface simulations of contact line motion, Phys. Rev. E 75 (2007) 046708.

[15] S. Afkhami, M. Bussmann, Height functions for applying contact angles to 3D VOF simulations, Int. J. Numer. Meth. Fluids 61 (2009) 827.

[16] S. Shin, J. Chergui, D. Juric, Direct simulation of multiphase flows with modeling of dynamic interface contact angle, Theor. Comput. Fluid Dyn. 32 (2018) 655 .

[17] P. Yue, Thermodynamically consistent phase-field modelling of contact angle hysteresis, J. Fluid Mech. 899 (2020) A15.

[18] H.-L. Li, H.-R. Liu, H. Ding, A fully 3D simulation of fluid-structure interaction with dynamic wetting and contact angle hysteresis, J. Comput. Phys. 420 (2020) 109709.

[19] T.-Y. Han, J. Zhang, H. Tan, M.-J. Ni, A consistent and parallelized height function based scheme for applying contact angle to 3D volume-of-fluid simulations, J. Comput. Phys. 433 (2021) 110190. 
[20] A. O'Brien, M. Bussmann, A moving immersed boundary method for simulating particle interactions at fluid-fluid interfaces, J. Comput. Phys. 402 (2020) 109089.

[21] S. Popinet, The Gerris flow solver, http://gfs.sourceforge.net/, 1.3.2 (2012).

[22] S. Popinet, Gerris: a tree-based adaptive solver for the incompressible Euler equations in complex geometries, J. Comput. Phys. 190 (2003) 572.

[23] S. Popinet, An accurate adaptive solver for surface-tension-driven interfacial flows, J. Comput. Phys. 228 (2009) 5838.

[24] S. Popinet, Numerical models of surface tension, Ann. Rev. Fluid Mech. 50 (2018) 49.

[25] S. Popinet, Basilisk, a free-software program for the solution of partial differential equations on adaptive Cartesian meshes, http://basilisk.fr (2018).

[26] T. Fullana, S. Zaleski, S. Popinet, Dynamic wetting failure in curtain coating by the Volume-of-Fluid method, Eur. Phys. J. Special Topics 229 (2020) 1923.

[27] J. Sakakeeny, Y. Ling, Numerical study of natural oscillations of supported drops with free and pinned contact lines, Phys. Fluids 33 (2021) 062109.

[28] T. D. Blake, The physics of moving wetting lines, J. Colloid Interface Sci. 299 (2006) 1.

[29] Y. Shikhmurzaev, Moving contact lines in liquid/liquid/solid systems, J. Fluid Mech. 334 (1997) 211.

[30] C. Huh, L. E. Scriven, Hydrodynamic model of steady movement of a solid/liquid/fluid contact line, J. Colloid Interface Sci. 35 (1971) 85.

[31] A. Dziedzic, M. Nakrani, B. Ezra, M. Syed, S. Popinet, S. Afkhami, Breakup of finite-size liquid filaments: Transition from no-breakup to breakup including substrate effects, Eur. Phys. J. E 42 (2019) 18.

[32] H. Liu, S. Krishnan, S. Marella, H. Udaykumar, Sharp interface Cartesian grid method II: A technique for simulating droplet interactions with surfaces of arbitrary shape, J. Comput. Phys. 210 (2005) 32.

[33] J. Zhang, P. Yue, A level-set method for moving contact lines with contact angle hysteresis, J. Comput. Phys. 418 (2020) 109636.

[34] J. W. Cahn, J. E. Hilliard, Free energy of a nonuniform system. 1. Interfacial free energy, J. Chem. Phys. 28 (1958) 258. 
[35] J. Jacqmin, Calculation of two-phase navier-stokes flows using phase field modeling, J. Comp. Phys. 155 (1999) 96.

[36] J. Jacqmin, Onset of wetting failure in liquid-liquid systems, J. Fluid Mech. 517 (2004) 209.

[37] P. Yue, C. Zhou, J. Feng, Sharp-interface limit of the Cahn-Hilliard model for moving contact lines, J. Fluid Mech. 645 (2010) 279.

[38] D. Sibley, A. Nold, S. Kalliadasis, Unifying binary fluid diffuse-interface models in the sharp interface limit, J. Fluid Mech. 736 (2013) 5.

[39] A. J. Briant, A. J. Wagner, J. M. Yeomans, Lattice boltzmann simulations of contact line motion. i. liquid-gas systems, Phys. Rev. E. 69 (2004) 031602 .

[40] T. Lee, L. Liu, Lattice boltzmann simulations of micron-scale drop impact on dry surfaces, J. Comp. Phys. 229 (2010) 8045.

[41] T. Qian, X.-P. Wang, P. Sheng, Molecular scale contact line hydrodynamics of immiscible flows, Phys. Rev. E 68 (2003) 016306.

[42] T. Qian, X.-P. Wang, P. Sheng, Molecular hydrodynamics of the moving contact line in two-phase immiscible flows, Comm. Comput. Phys. 1 (2006) 1.

[43] T. Nguyen, M. Fuentes-Cabrera, J. Fowlkes, J. Diez, A. González, L. Kondic, P. Rack, Competition between collapse and breakup in nanometer-sized thin rings using molecular dynamics and continuum modeling, Langmuir 28 (2012) 13960.

[44] M. Fuentes-Cabrera, B. Rhodes, J. Fowlkes, A. López-Benzanilla, H. Terrones, M. Simpson, P. Rack, Molecular dynamics study of the dewetting of copper on graphite and graphene: Implications for nanoscale selfassembly, Phys. Rev. E 83 (2011) 041603.

[45] S. Afkhami, L. Kondic, Numerical simulation of ejected molten metal nanoparticles liquified by laser irradiation: Interplay of geometry and dewetting, Phys. Rev. Lett. 111 (2013) 034501.

[46] U. Lăcis, P. Johansson, T. Fullana, B. Hess, G. Amberg, S. Bagheri, and S. Zaleski, Steady moving contact line of water over a no-slip substrate, Eur. Phys. J. Special Topics 229 (2020) 1897.

[47] W. Ren, W. E, Heterogeneous multiscale method for the modeling of complex fluids and micro-fluidics, J. Comput. Phys. 204 (2005) 1.

[48] H. Huang, D. Liang, B. B. Wetton, Computation of a moving drop/bubble on a slid surface using a front-tracking method, Commun. Math. Sci. 2 (2004) 535. 
[49] K. Mahady, S. Afkhami, L. Kondic, A volume of fluid method for simulating fluid/fluid interfaces in contact with solid boundaries, J. Comp. Phys. 294 (2015) 243.

[50] J. U. Brackbill, D. B. Kothe, C. Zemach, A continuum method for modeling surface tension, J. Comput. Phys. 100 (1992) 335.

[51] K. Mahady, S. Afkhami, L. Kondic, A numerical approach for the direct computation of flows including fluid-solid interaction: modeling contact angle, film rupture, and dewetting, Phys. Fluids 28 (2016) 062002.

[52] R. G. Cox, The dynamics of the spreading of liquids on a solid surface. Part 1. Viscous flow, J. Fluid Mech. 168 (1986) 169.

[53] O. Voinov, Hydrodynamics of wetting, Fluid Dynamics 11 (1976) 714.

[54] D. Bonn, J. Eggers, J. Indekeu, J. Meunier, E. Rolley, Wetting and spreading, Rev. Mod. Phys. 81 (2009) 739.

[55] R. Seemann, S. Herminghaus, K. Jacobs, Gaining control of pattern formation of dewetting liquid films, J. Phys. Condens. Matt. 21 (2001) 4925.

[56] C. Neto, K. Jacobs, R. Seemann, R. Blossey, J. Becker, G. Grün, Satellite hole formation during dewetting: experiment and simulation, J. Phys.: Condens. Matter 15 (2003) 3355.

[57] J. Becker, G. Grün, R. Seemann, H. Mantz, K. Jacobs, K. R. Mecke, R. Blossey, Complex dewetting scenarios captured by thin-film models, Nature Mat. 2 (2003) 59.

[58] Y. Oh, J. Lee, M. Lee, Fabrication of Ag-Au bimetallic nanoparticles by laser-induced dewetting of bilayer films, Appl. Surf. Sci. 434 (2018) 1293.

[59] D. A. Garfinkel, G. Pakeltis, N. Tang, I. N. Ivanov, J. D. Fowlkes, D. A. G., P. D. Rack, Optical and magnetic properties of ag-ni bimetallic nanoparticles assembled via pulsed laser-induced dewetting, ACS Omega 5 (2020) 19285 .

[60] M. Fricke, D. Bothe, Boundary conditions for dynamic wetting - A mathematical analysis, Eur. Phys. J. Special Topics 229 (2020) 1849.

[61] S. Afkhami, T. Gambaryan-Roisman, L. M. Pismen, Challenges in nanoscale physics of wetting phenomena, Eur. Phys. J. Special Topics 229 (2020) 1735.

[62] J. H. Snoeijer, B. Andreotti, Moving contact lines: Scales, regimes, and dynamical transitions, Annu. Rev. Fluid Mech. 45 (2013) 269.

[63] G. Tryggvason, R. Scardovelli, S. Zaleski, Direct Numerical Simulations of Gas-Liquid Multiphase Flows, Cambridge University Press, 2011. 
[64] L. D. Landau, B. V. Levich, Dragging of a liquid by a moving plate, Acta Physicochim. URSS 17 (1942) 42.

[65] B. V. Derjaguin, On the thickness of a layer of liquid remaining on the walls of vessels after their emptying, and the theory of the application of photoemulsion after coating on the cine film, Acta Physicochim. URSS 20 (1943) 349.

[66] J. Eggers, Hydrodynamic theory of forced dewetting, Phys. Rev. Lett. 93 (2004) 094502.

[67] J. Eggers, Contact line motion for partially wetting fluids, Phys. Rev. E 72 (2005) 061605.

[68] T. S. Chan, J. H. Snoeijer, J. Eggers, Theory of the forced wetting transition, Phys. Fluids 24 (2012) 072104.

[69] D. Legendre, M. Maglio, Comparison between numerical models for the simulation of moving contact lines, Comput. Fluids 113 (2015) 2.

[70] S. Afkhami, J. Buongiorno, A. Guion, S. Popinet, Y. Saade, R. Scardovelli, S. Zaleski, Corrigendum to "Transition in a numerical model of contact line dynamics and forced dewetting" [J. Comput. Phys. 374(2018)10611093], J. Comput. Phys. 382 (2019) 61.

[71] J. Qin, P. Gao, Asymptotic theory of fluid entrainment in dip coating, J. Fluid Mech. 844 (2018) 1026.

[72] J. A. Moriarty, L. W. Schwartz, Effective slip in numerical calculations of moving-contact-line problems, J. Eng. Math. 26 (1992) 81.

[73] O. Weinstein, L. M. Pismen, Scale dependence of contact line computations, Math. Model. Nat. Phenom. 3 (2008) 98.

[74] P. Yue, J. J. Feng, Wall energy relaxation in the Cahn-Hilliard model for moving contact lines, Phys. Fluids 23 (2011) 012106.

[75] T. Blake, M. Bracke, Y. Shikhmurzaev, Experimental evidence of nonlocal hydrodynamic influence on the dynamic contact angle, Phys. Fluids 11 (1999) 1995.

[76] M. Wilson, J. Summers, Y. Shikhmurzaev, A. Clarke, T. Blake, Nonlocal hydrodynamic influence on the dynamic contact angle: Slip models versus experiment, Phys. Rev. E 73 (2006) 041606.

[77] T. Qian, X.-P. Wang, P. Sheng, Generalized Navier boundary condition for the moving contact line, Commun. Math. Sci. 1 (2003) 333.

[78] M. Fricke, M. Köhne, D. Bothe, A kinematic evolution equation for the dynamic contact angle and some consequences, Phys. D: Nonlinear Phenom. 394 (2019) 26. 
[79] X.-P. Wang, T. Qian, P. Sheng, Moving contact line on chemically patterned surfaces, J. Fluid Mech. 605 (2008) 59.

[80] X. Xu, X.-P. Wang, Analysis of wetting and contact angle hysteresis on chemically patterned surfaces, SIAM J. Appl. Math. 71 (2011) 1753.

[81] W. Ren, Wetting transition on patterned surfaces: Transition states and energy barriers, Langmuir 30 (2014) 2879.

[82] L. Luo, X.-P. Wang, X.-C. Cai, An efficient finite element method for simulation of droplet spreading on a topologically rough surface, J. Comput. Phys. 349 (2017) 233.

[83] W. Li, J. Lu, G. Tryggvason, Y. Zhang, Numerical study of droplet motion on discontinuous wetting gradient surface with rough strip, Phys. Fluids 33 (2021) 012111.

[84] A. Ferrari, I. Lunati, Direct numerical simulations of interface dynamics to link capillary pressure and total surface energy, Adv. Water Resour. 57 (2013) 19.

[85] A. Q. Raeini, M. J. Blunt, B. Bijeljic, Direct simulations of two-phase flow on micro-ct images of porous media and upscaling of pore-scale forces, Adv. Water Resour. 57 (2014) 116.

[86] S. Bakhshian, S. Hosseini, N. Shokri, Pore-scale characteristics of multiphase flow in heterogeneous porous media using the lattice Boltzmann method, Adv. Water Resour. 9 (2019) 3377.

[87] B. Zhao, C. W. MacMinn, B. K. e. a. Primkulov, Comprehensive comparison of pore-scale models for multiphase flow in porous media, Proc. Natl. Acad. Sci. 116 (28) (2019) 13799.

[88] N. Ghillani, M. Heinz, T. Gambaryan-Roisman, Capillary rise and evaporation of a liquid in a corner between a plane and a cylinder: A model of imbibition into a nanofiber mat coating, Eur. Phys. J. Special Topics 229 (2020) 1799.

[89] F. Basirat, Z. Yang, A. Niemi, Pore-scale modeling of wettability effects on $\mathrm{CO}_{2}$-brine displacement during geological storage, Adv. Water Resour. 109 (2017) 181.

[90] H. Liu, A. J. Valocchi, C. Werth, Q. Kang, M. Oostrom, Pore-scale simulation of liquid $\mathrm{CO}_{2}$ displacement of water using a two-phase lattice Boltzmann model, Adv. Water Resour. 73 (2014) 144.

[91] C. S. Peskin, Flow patterns around heart valves: A numerical method, J. Comput. Phys. 10 (1972) 252.

[92] R. Mittal, G. Iaccarino, Immersed Boundary Methods, Annu. Rev. Fluid Mech. 37 (2005) 239. 
[93] A. O'Brien, M. Bussmann, A volume-of-fluid ghost-cell immersed boundary method for multiphase flows with contact line dynamics, Comput. \& Fluids 165 (2018) 43.

[94] A. O'Brien and S. Afkhami and M. Bussmann, Pore-scale direct numerical simulation of Haines jumps in a porous media model, Eur. Phys. J. Special Topics 229 (2020) 1785.

[95] S. G. Kandlikar, History, Advances, and Challenges in Liquid Flow and Flow Boiling Heat Transfer in Microchannels: A Critical Review, J. Heat Transfer 134.

[96] C. Kunkelmann, K. Ibrahem, N. Schweizer, S. Herbert, P. Stephan, T. Gambaryan-Roisman, The effect of three-phase contact line speed on local evaporative heat transfer: Experimental and numerical investigations, Int. J. Heat Mass Transfer 55 (2012) 1896.

[97] A. Guion, S. Afkhami, S. Zaleski, J. Buongiorno, Simulations of microlayer formation in nucleate boiling, Int. J. Heat Mass Transfer 127 (2018) 1271.

[98] K. Schweikert, A. Sielaff, P. Stephan, On the transition between contact line evaporation and microlayer evaporation during the dewetting of a superheated wall, Int. J. Thermal Sci. 145 (2019) 106025.

[99] L. Bureš, Y. Sato, On the modelling of the transition between contact-line and microlayer evaporation regimes in nucleate boiling, J. Fluid Mech. 916 (2021) A53.

[100] I. Seric, S. Afkhami, L. Kondic, Influence of thermal effects on stability of nanoscale films and filaments on thermally conductive substrates, Phys. Fluids 30 (2018) 012109. 